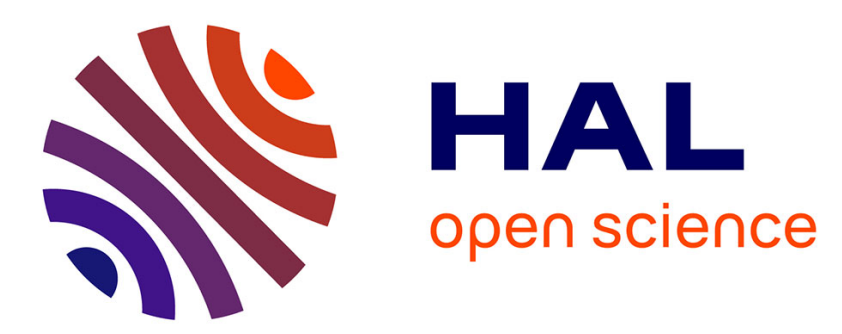

\title{
Dynamic Lifting Motion of Humanoid Robots
}

Hitoshi Arisumi, Jean-Rémy Chardonnet, Abderrahmane Kheddar, Kazuhito

Yokoi

\section{To cite this version:}

Hitoshi Arisumi, Jean-Rémy Chardonnet, Abderrahmane Kheddar, Kazuhito Yokoi. Dynamic Lifting Motion of Humanoid Robots. ICRA'07: International Conference on Robotics and Automation, Apr 2007, Rome, Italy. pp.2661-2667. lirmm-00536325

\section{HAL Id: lirmm-00536325 https://hal-lirmm.ccsd.cnrs.fr/lirmm-00536325}

Submitted on 15 Nov 2010

HAL is a multi-disciplinary open access archive for the deposit and dissemination of scientific research documents, whether they are published or not. The documents may come from teaching and research institutions in France or abroad, or from public or private research centers.
L'archive ouverte pluridisciplinaire HAL, est destinée au dépôt et à la diffusion de documents scientifiques de niveau recherche, publiés ou non, émanant des établissements d'enseignement et de recherche français ou étrangers, des laboratoires publics ou privés. 


\title{
Dynamic Lifting Motion of Humanoid Robots
}

\author{
Hitoshi Arisumi, Jean-Rémy Chardonnet, \\ Abderrahmane Kheddar, Member, IEEE, and Kazuhito Yokoi, Member, IEEE
}

\begin{abstract}
This paper describes a motion generation method for dynamic lifting by a humanoid robot. The proposed technique suggests the possibility of taking advantage of the whole body motion in order to facilitate the lifting movement. In particular, the idea is to perform a preliminary motion in order to generate a momentum which is instantaneously transferred to the object as an impulsive force. This allows the humanoid to lift up an object that could not be lifted up only by continuous force. However an impulsive force may make the humanoid unstable. Then, we propose to set the center of percussion (CoPn) of the whole system at the center of the support polygon of the humanoid when it lifts up the object. We also propose a design method of a preliminary motion of the humanoid that generates a sufficient momentum to lift up an object without any slip, tumble and hop of the whole system. The effectiveness of the proposed method is confirmed by simulation and experiment.
\end{abstract}

Index Terms-Humanoid robot, Dynamic whole body manipulation, ZMP, Centre of percussion, Momentum transfer

\section{INTRODUCTION}

\section{A. Background}

$\mathrm{H}$ UMANS often generate preliminary motion and use it for another succeeding motion such as manipulation of an object in daily life. For instance, before throwing a ball or opening the door that has been shut fast, we generate a whole body motion to apply a larger force to the object. It would appear that there are two effects of the preliminary motion. One is the generation of auxiliary forces. When we can not move a heavy object by a continuous force as shown in Fig. 1(a), we enlarge the kinetic momentum of our own body by a preliminary motion and try to use it for applying impulsive force to the object. The other effect is to avoid unstable states such as releasing heel or toe from the floor. If the ground projection of the center of total mass (GCoM) of both an object and us is located ahead of our feet as shown in Fig. 1(b), we can not manipulate the object by a continuous force

Manuscript received September 15, 2006; revised January 30, 2007. This work was supported in part by the Japan Society for the Promotion of Science (JSPS) under Grants-in-Aid for Scientific Research.

H. Arisumi and K. Yokoi are with the National Institute of Advanced Industrial Science and Technology (AIST), AIST Central 2, 1-1-1 Umezono, Tsukuba, Ibaraki, 305-8568 Japan (e-mail: h-arisumi@aist.go.jp; kazuhito.yokoi@aist.go.jp).

J-R. Chardonnet is with the LIRMM and the AIST-CNRS Joint Japanese -French Robotics Laboratory (JRL) (e-mail: jr-chardonnet@aist.go.jp).

A. Kheddar is with the AIST-CNRS Joint Japanese-French Robotics Laboratory (JRL) (e-mail: abderrahmane.kheddar@aist.go.jp). because our feet are not fixed in the environment. No matter how high power we have, our body leans forward around the toe in this case. But utilizing a kinetic momentum enlarged by a preliminary motion, we can lift the object without tumbling. It can be said that preliminary motion gives us higher physical capabilities.

On the other hand, preliminary motion for manipulation of unfixed mount type robot such as humanoid robot is not used in previous works. Manipulation by a robot has been especially discussed only in the case where the GCoM of the robot and an object is located within the support polygon formed by the outside edges of robot feet at the first moment [6]. But in usual tasks, a robot can not always put the GCoM in the support area at the beginning of a manipulation. Fig. 2 illustrates cases where a robot can not stand sufficiently close to an object due to an obstacle or a hole between the robot and the object, or due to the shape of the object. It is quite difficult to lift the object by the previous method in these cases corresponding to the case shown in Fig. 1(b).

If useful skills of the humans using preliminary motion can be applied to robot manipulation, we can expect that feasible tasks of robots will be extended.

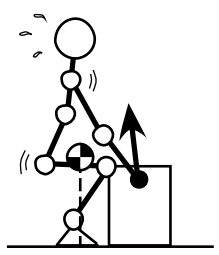

(a)

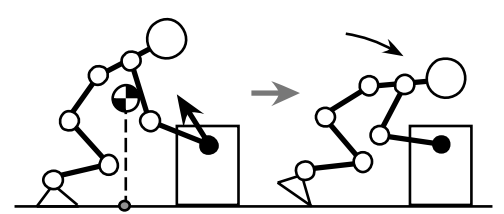

(b)
Fig. 1. Infeasible case of lifting by continuous force. (a) Powerless lifting. (b) Leaning forward with heel up.
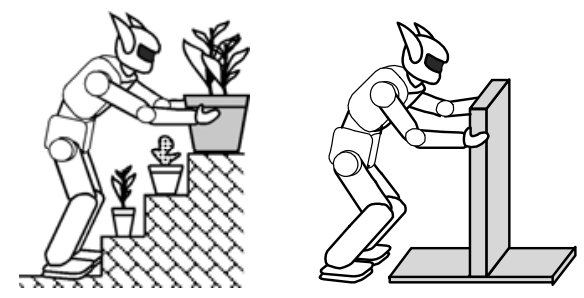

Fig. 2. Situations where a robot can not get closer to an object.

\section{B. Related Works}

Dynamic manipulation of fixed manipulator has been keenly discussed. For example, rolling control of a box on the paddle of manipulator [1] or rolling control of a ball on the butterfly shaped robot arm [2] have been studied. Zhu proposed a releasing manipulation, in which an object is hit on a horizontal plane by a serial manipulator [3]. However it 
is not necessary to discuss tumble or stability of robot in these studies because the base of robot is fixed in environment.

To take an example discussed in manipulation of unfixed robot such as humanoid robots, cooperative transportation by a human and a robot [4], pushing manipulation or lifting motion have been studied. Takubo focused on pushing a cart controlled by a humanoid robot, and proposed a method of a pattern generation to satisfy both stability and pushing force by locating the CoM of the robot in the support area given by contact configuration of both legs and hands [5]. Harada realized a pushing manipulation by a humanoid robot based on position control and force control [6]. He also proposed a method of lifting an unknown object by controlling the posture of the humanoid robot to locate a static balancing point in the support area [7]. But manipulation of an object in unstable state as shown in Fig. 2 is not considered in these studies. Konno dealt with the whole body motion of the humanoid and discussed appropriate working postures to apply a force to an environment [8]. But the dynamical effect is not considered in this paper.

Lifting motion may be likened to a kicking motion by comparing hind leg, its foot and fore leg to arm, object and supporting leg respectively. The weight of the robot foot is not well considered in previous works on kicking motion. It is regarded as disturbance in kicking control based on pendulum model or cart-table model. Study on walking control of a biped robot focused mainly on horizontal motion of the CoM, but vertical motion as well as rotational motion around the waist should be also discussed.

As for study on human lifting motion, analysis of torque pattern of expert lifter [9] or lifting speed of amateur lifter [10] are reported. But it may be difficult to apply these analytical results of complicate and agile motion effectively for robotic motion, because the mechanical structure or actuator-characteristics of robot are different from those of human-being.

\section{Research Target}

In this paper, we focus on lifting motion from the initial state shown in Fig. 1(b) where the GCoM of the robot and the object is outside of the foot area. Comparing with previous method of lifting, in which the robot lifts an object by continuous force from static initial state, we discuss effects of preliminary motion for lifting task and stability.

\section{FORMULATION}

\section{A. Model}

We here assume that lifting motion is a symmetric motion of robot and object. For our purpose, we take a 7-dof system in the sagittal plane as a model of robot. As shown in Fig. 3, weights of the robot and the object are denoted as mrob and mobj respectively, and the moment of inertia around the CoM of the robot is denoted as $I_{r o b} . f_{h}$ and $\tau_{h}$ are the force and the torque applied to the hand respectively, $\left[x_{h}, z_{h}\right]^{\mathrm{T}}$ and $\left[x_{o b j}\right.$, $\left.z_{o b j}\right]^{\mathrm{T}}$ are the position of the handling point and the center of

the object in the object frame respectively. $d_{o b j}$ represents the length of the object base. $\boldsymbol{f}_{\text {foot }}=\left[f_{\text {foot }}, f_{\text {footz }}\right]^{\mathrm{T}}$ represents the force vector applied to the floor by the robot foot. $\mathrm{Ji}$ $(i=1,2, \ldots, 7)$ represents joint $i$.

Since the mass of the torso composed of waist, chest and head is the majority of total mass of the robot, its mass is regarded as that of the robot and its CoM is representative of that of the robot. We assume that the mass of the object is centralized on its CoM, and the grasping point is the CoM of the object in this paper.

\section{B. Static stable/unstable reachable area}

The static stable reachable area (SsRA) is defined as the set of positions of the robot's hands where the GCoM of the total mass of the robot and an object is inside the support polygon. In the SsRA, the robot can statically keep the position of its hand in some posture of the robot. When the robot is in the static state with its hand out of SsRA, the robot tumbles. The size and shape of SsRA varies with the load at the hand. As Fig. 4 illustrates schematically, the gray area indicates the SsRA when the hand is free from the load. When the robot grasps an object with some weight, there are two types of SsRA. One is the hatched area in Fig. 5 where the object is away from floor. The other is the horizontal line passing through the grasping point on the object when it is on the floor. The SsRA narrows and distributes closer to the vertical line passing through the ankle joint as the weight of the object gets bigger.

In this paper, we assume that the initial state and the final state are both static, and start point Ps and end point Pe of robot's hand are located on the horizontal line and in the hatched area in Fig. 5, respectively. In the static case of lifting, a continuous force can be applied to the robot's hand to follow the trajectory. However, the robot becomes unstable in this case. The question we have to ask here is how to lift an object from the initial state to the final state passing through an unstable state as shown in Fig. 5.
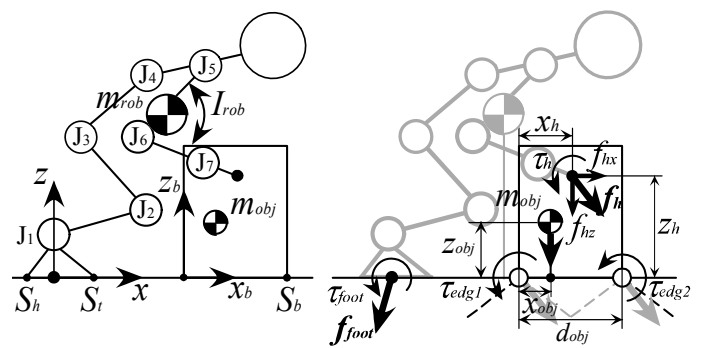

Fig. 3. Humanoid robot model.

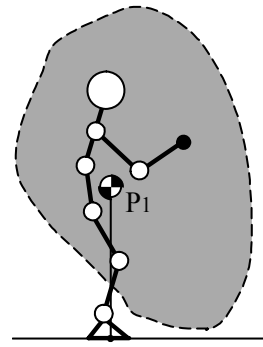

Fig. 4. Reachable area.

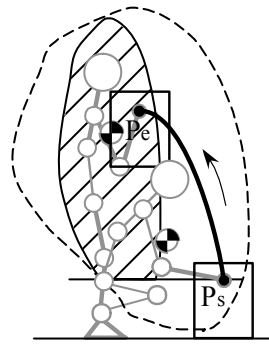

Fig. 5. Trajectory of hand.

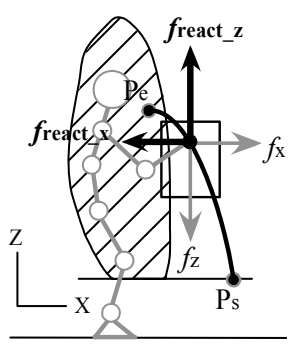

Fig. 6. Operational force. 


\section{Strategy for lifting}

We assume that the GCoM of the whole system at the initial state is inside the support area described as the region between $S_{h}$ and $S_{b}$ in Fig. 3, but not inside the foot area described as region between $S_{h}$ and $S_{t}$. Then the ZMP at the initial state is outside the foot area because the ZMP and the GCoM of the whole system are generally the same point when the robot and the object are in the static state. Consequently when the robot starts to lift an object by a continuous force from the static state, it is necessary to make the ZMP move instantly from the initial position to the foot area to avoid the robot tumbling. But in this case, the high power actuation for motion of the robot and the object is required. As a practical matter, it is quite difficult for the static robot to move the ZMP drastically.

Even if an object leaves the floor by some means, the robot needs to generate an inertial force to avoid tumble. As shown in Fig. 6, reaction forces $f_{\text {react_ } x}$ and $f_{\text {react_z }} z$ generated by pushing an object along the direction of $\mathrm{x}$-axis and the negative direction of $\mathrm{z}$-axis respectively can be used for inertial force. However pushing force $f_{x}$ and $f_{z}$ shown in Fig. 6 keep an object far away from end point $\mathrm{P}_{\mathrm{e}}$. Then it is quite difficult to move an object toward the end point by this way.

To solve these problems, we make use of an impulsive force as an auxiliary force for lifting motion and stability. We then focus on a preliminary motion to enlarge the kinetic momentum of the robot which is instantaneously transferred to the object as an impulsive force. The ZMP can be moved to the foot support area before the object leaves from the floor by the preliminary motion.

Fig. 7 illustrates sequential motion of lifting an object with preliminary motion. It consists of five phases, namely sitting, leaning forward, raising upper body, the pulling, and holding an object. The phases proceed from the left to the right. Motion from leaning forward to raising upper body corresponds to the preliminary motion.

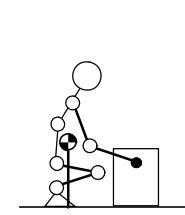

(a)

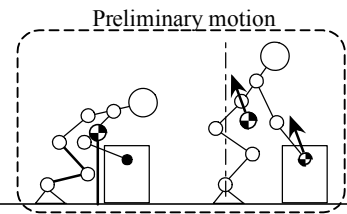

(b)

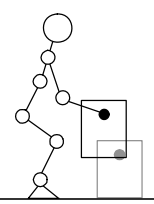

(d)

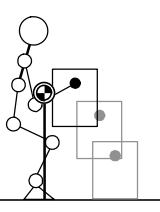

(e)
Fig. 7. Lifting motion of robot by impact. (a) Sitting. (b) Leaning forward. (c) Raising. (d) Pulling. (e) Holding.

\section{LIFTING WITH PRELIMINARY MOTION}

Since humanoid robot is highly redundant, there may be many trajectories which realize lifting motion shown in Fig. 7. We discuss the lifting motion by dividing it into three events, namely motions before/at/after the impact in this section.

Fig. 8 illustrates an image of impact lifting which we propose. As shown in Fig. 8(a), the robot enlarges its momentum before lifting, and the momentum is transferred to an object at the impact. Then the object is lifted with the robot as a translational motion. But the robot has to move upward to lift the object to a higher position in a translational motion. It is an inefficient way in terms of energy consumption to move the robot defying gravity. For this reason, we focus on a rotational motion around the center of foot at the impact as shown in Fig. 8(b). It is generated by transformation of motion. We thus use the inverted pendulum model for rotational motion after impact.

Motions before/at/after the impact correspond to preliminary motion, impact motion, and inverted pendulum motion respectively. We discuss them in reverse order.

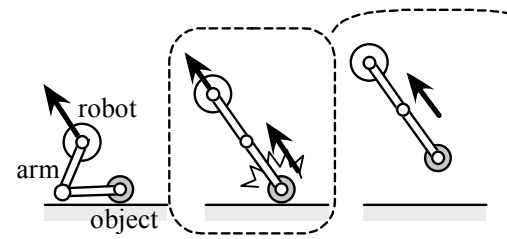

(a)

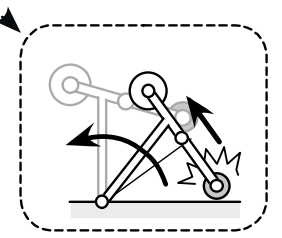

(b)
Fig. 8. Image of impact lifting. (a) Translational motion before/at/after impact. (b) Rotational motion.

\section{A. Inverted pendulum motion}

Fig. 9 shows an inverted pendulum model for control of the robot and the object after impact. "A" in Fig. 9 represents the CoM of the whole system, namely the torso of the robot and the object. "B" represents the center of the foot. We denote the mass of the whole system as mall, its inertia momentum around the center B as IallB. We here assume the followings:

- After impact, configuration of the upper body is fixed

- Position of the CoM of the whole system (point A) is controlled by the legs to follow the inverted pendulum motion around the point $\mathrm{B}$

- Target position of the point $A$ is on the line of intersection of the vertical plane passing through both ankle joints and the symmetrical plane for the robot

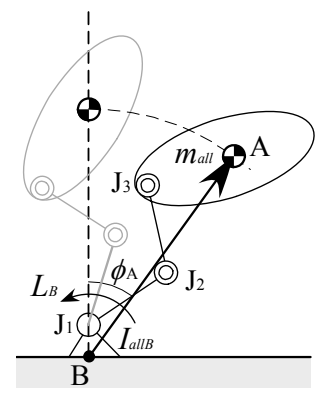

Fig. 9. Inverted pendulum model.

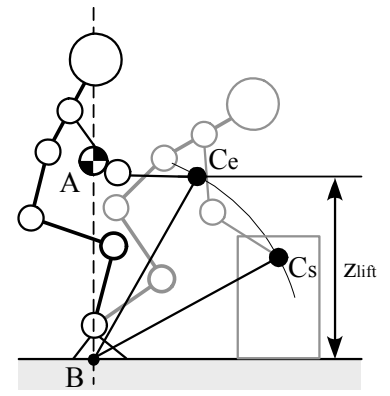

Fig. 10. Lifting height.
A state of the robot at the impact corresponds to an initial state of the inverted pendulum. Giving the initial position of hand (point $\mathrm{Ce}$ ) and the lifting height $z_{\text {liff }}$, the intersection of circular orbit and the horizontal line at the height of $z_{\text {lift }}$ is the final position of the hand (point $\mathrm{Cs}_{\mathrm{s}}$ ) as shown in Fig. 10.

The point to be discussed here is to estimate the angular momentum just after impact which makes the robot reach a statically stable state with the object. The target point corresponds to the equilibrium point of the inverted pendulum. By using mechanical energy function expressed in terms of the generalized coordinate, the angular momentum $L_{B}$ of the whole system can be given by 


$$
L_{B}=\operatorname{sqrt}\left(2 \text { IallB mall } g\left|\boldsymbol{r}_{A B}\right|\left(1-\cos \phi_{\mathrm{A}}\right)\right),
$$

where $\boldsymbol{r}_{j k}$ is a vector from point $\mathrm{j}$ to point $\mathrm{k}$ in the frame $\Sigma \mathrm{xz}$, $\left|\boldsymbol{r}_{j k}\right|$ is the distance between the point $\mathrm{j}$ to the point $\mathrm{k}$, and $\phi_{\mathrm{A}}$ is the angle between the vertical line and the line $\mathrm{AB}$ at the initial state. IallB $=$ Iall + mall $\left|\boldsymbol{r}_{A B}\right|^{2}$.

\section{B. Impact motion}

Impact is used for lifting, but meanwhile it can make the system unstable. When the robot applies an impulse to an object for lifting it, the robot can receive a large reaction force in a short time. At that time, each part of the robot is accelerated and velocity of it can be instantaneously changed by the impact. In this case it is necessary to prevent the foot from moving because movement of the foot possibly makes the robot unstable.

We here focus on the center of percussion ( $\mathrm{CoPn})$ to reduce the negative effect of impact. The CoPn is the point where an impact will produce translational and rotational forces which perfectly cancel each other [11]. In other words, translational and rotational velocities generated by impact perfectly cancel each other at the point. Then the $\mathrm{CoPn}$ is the pivot point where velocity does not change when impact occurs. We propose to set the CoPn at the center of the support polygon of the robot when the robot lifts up the object. We investigate motion of the robot before/after impact to set the CoPn at the center of the foot.

As shown in Fig. 11, "C" $\left[x_{c}, z_{c}\right]^{\mathrm{T}}$ is the CoM of the object, " $\mathrm{D}$ " $\left[x_{r o b}, z_{r o b}\right]^{\mathrm{T}}$ is the CoM of the robot. Line $l_{1}$ represents a lifting direction passing through the point $\mathrm{C}, \varphi$ is the lifting angle between line $l_{1}$ and the horizontal line. Line $l_{2}$ and line $l_{3}$ are parallel to line $l_{1}$ and passing through the point $\mathrm{A}$ and $\mathrm{D}$ respectively. ${ }^{*} \mathrm{z}$ is the axis of a frame $\Sigma^{*} \mathrm{x} * \mathrm{z}$ parallel to line $l_{1}$, $l_{2}$ and $l_{3}$. "E" is the foot of the perpendicular to the line $l_{1}$ from the point B. "F" and "G" are the intersections of line BE and line $l_{2}$, and line $l_{3}$ respectively. When we consider the whole system as a single body, we denote its mass as mall $\left(=m_{r o b}+m_{o b j}\right)$, the velocity vector of its center $\mathrm{A}$ as $\boldsymbol{v}_{\text {all }}=[$ vallx, $\left.v_{\text {allz }}\right]^{\mathrm{T}}$, its counter clockwise angular velocity as wall and the inertia momentum around the center A as Iall respectively. We also denote velocities before and after lifting the object as *v and ${ }^{*} \underline{v}$ respectively in the frame $\Sigma^{*} \mathrm{x}^{*} \mathrm{z}$.

Supposing that external torque does not apply to the whole system when impact occurs, we get the following equation from the law of conservation of angular momentum around the point A:

$$
\begin{aligned}
& I_{r o b} * \omega r o b+* \boldsymbol{r}_{A D} \times m_{r o b} * \boldsymbol{v}_{r o b}+* \boldsymbol{r}_{A C} \times m_{o b j} * \boldsymbol{v}_{o b j} \\
& \quad=I_{a l l} * \underline{\omega a l l}^{*}+\boldsymbol{r}_{A D} \times m_{r o b} * \underline{\boldsymbol{v}} r o b+* \boldsymbol{r}_{A C} \times m_{o b j} * \underline{\boldsymbol{v}} o b j
\end{aligned}
$$

where ${ }^{*} \boldsymbol{r}_{A D}=\left[{ }^{*} r_{A D x},{ }^{*} r_{A D}\right]^{\mathrm{T}}$ is the vector from the point $\mathrm{A}$ to the point $\mathrm{D}$ in the frame $\Sigma^{*} \mathrm{x} * \mathrm{z},{ }^{*} \boldsymbol{r}_{A C}=\left[{ }^{*} r_{A C X},{ }^{*} r_{A C Z}\right]^{\mathrm{T}}$ is the vector from the point $\mathrm{A}$ and the point $\mathrm{C}$ in the same frame. ${ }^{*} \boldsymbol{v}_{r o b}=$ $\left[{ }^{*} v_{r o b x}, * v_{r o b z}\right]^{\mathrm{T}}$ is the velocity vector of the robot and $* \boldsymbol{v}_{o b j}=$ $\left[{ }^{*} v_{o b j x},{ }^{*} v_{o b j z}\right]^{\mathrm{T}}$ is that of the object. " $X$ " in (2) represents the cross product. Here we assume the following things:
- The object is static before impact $\left({ }^{*} v_{o b j x}=* v_{o b j z}=0\right)$

- The robot does not move in the direction of $*_{\mathrm{x}}$ axis before impact $\left(*^{*} v_{r o b x}=0\right)$

- The robot and the object are regarded as a single body after impact $\left({ }^{*} \underline{v}_{r o b x}={ }^{*} \underline{v}_{o b j x}={ }^{*} \underline{v} a l l x,{ }^{*} \underline{v}_{r o b z}={ }^{*} \underline{v}_{o b j z}={ }^{*} \underline{v a l l}\right)$

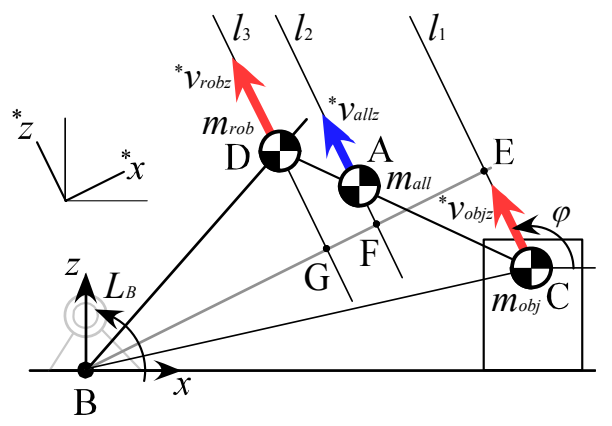

Fig. 11. Position and velocity of CoM when lifting.

Since the robot applies impulse to the object in the direction of $*_{z}$ axis, velocity of the robot and the object after impact in the direction of $* \mathrm{x}$ axis does not change. Hence, ${ }^{*} \underline{v}_{r o b x}={ }^{*} \underline{v}_{o b j x}$ $=\underline{v}^{\text {vall }} x=0$. As the point $\mathrm{A}$ divides segment $\mathrm{CD}$ in the ratio of mass, ${ }^{*} r_{A D x}=-{ }^{*} r_{A C x} m_{o b j} / m_{r o b}$. Then considering assumptions described above, (2) can be simplified as follows:

$$
* \omega_{r o b}=\left(I_{a l l} * \underline{\omega}_{a l l}+* r_{A c_{x}} m_{o b j} * v_{r o b z}\right) / \text { Irob }
$$

Assuming that the point $\mathrm{B}$ is the CoPn, velocity of the robot at the point $\mathrm{B}$ does not change before/after impact. We obtain a condition described by the following equation [11]:

$$
{ }^{*} \underline{v} \text { allz_B } B={ }^{*} \underline{v} \text { allz }-\left|{ }^{*} \boldsymbol{r}_{B F}\right| * \underline{\omega} \text { all }=0
$$

where $*_{\text {vall } B} B$ is velocity of robot at the point $B$. Since the equation of line $l_{1}$ is expressed by $x \tan \varphi-z-x_{C} \tan \varphi+z_{C}=0$, line $l_{1}$ is uniquely identified when giving values of both $\varphi$ and $\left[x_{c}, z_{c}\right]^{\mathrm{T}}$. Then the shortest distance from the point $\mathrm{B}$ to line $l_{1}$, which is represented by $\left|{ }^{*} \boldsymbol{r}_{B E}\right|$, is given by

$$
\left|\boldsymbol{r}_{B E}\right|=\left|-x_{C} \tan \varphi+z_{C}\right| / \operatorname{sqrt}\left(\tan ^{2} \varphi+1\right)
$$

According to the geometrical conditions, we get followings:

$$
\left|\boldsymbol{r}_{B F}\right|=\left|{ }^{*} \boldsymbol{r}_{B E}\right|-\left|{ }^{*} \boldsymbol{r}_{F E}\right|=\left|\boldsymbol{r}_{B E}\right|-\left|{ }^{*} r_{A C X}\right| .
$$

When angular momentum around the point B after impact $L B$ is given by (1), the velocity of whole system should be satisfied with the following equation.

$$
L_{B}=\left|{ }^{*} \boldsymbol{r}_{B F}\right| \text { mall } \underline{v}_{\text {allz }}+I_{\text {all }} * \underline{\omega} \text { all }
$$

Once the configuration of robot at the impact is given, $I_{r o b}$ and Iall are calculated. The following equation is given by (4) and (7):

$$
{ }^{*} \underline{\omega} \text { all }=L B /\left(\left|\boldsymbol{r}_{B F}\right|^{2} \text { mall }+ \text { Iall }\right)
$$


Substituting (8) to (3), we obtain the relation between *vrobz and * ${ }^{*}$ rob.

Absolute coordinates of velocities of CoMrob are calculated by coordinate transformation $\left(\Sigma^{*}->\Sigma\right)$ as follows:

$$
\boldsymbol{V}_{r o b}=\boldsymbol{\operatorname { R o t }}(\varphi) * \boldsymbol{V}_{r o b}
$$

where $\boldsymbol{V}_{r o b}=\left[v_{r o b x}, v_{r o b z}, \omega_{r o b}\right]^{\mathrm{T}}, * \boldsymbol{V}_{r o b}=\left[{ }^{*} v_{r o b x},{ }^{*} v_{r o b z}, * \omega_{r o b}\right]^{\mathrm{T}}$ and $\boldsymbol{R o t}(*)$ is the rotation matrix.

\section{Preliminary motion}

In previous paragraph, we clarified the relation between $*^{*}$ robz and * ${ }^{*}$ rob which reduces negative effect of impact. We here discuss how to design preliminary motion considering the relation. For simplification of this subject, we assume the following things:

- The power of the actuators of the arms is small. Then the arms are nearly stretched when impact occurs in order not to yield to impulsive force in the direction of the stretched arm.

- Angle of joints 4 and 7 in Fig. 3 are constant

- Position of the ankle and the wrist are constant

Under these conditions, all joint angles of the robot are calculated by the inverse kinematics when the position and posture of torso are given. Then we design the trajectory of the position and posture of the torso to obtain the desired joint angles which are command data.

Giving the position of the object, the foot of the robot, and the direction of lifting the object, we first decide a path for the CoMrob in the preliminary motion. Here we suppose that the path is parallel to the lifting direction for simplicity. Then as shown in Fig. 12 the path is obtained by giving an initial position of the CoMrob (point $\mathrm{D}_{0}$ ) considering the geometrical conditions. Moreover the impact position of the CoMrob on the path (point D) is decided by considering the stretched arm and the inverted pendulum motion.

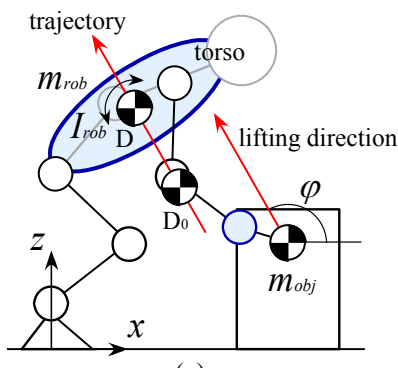

(a)

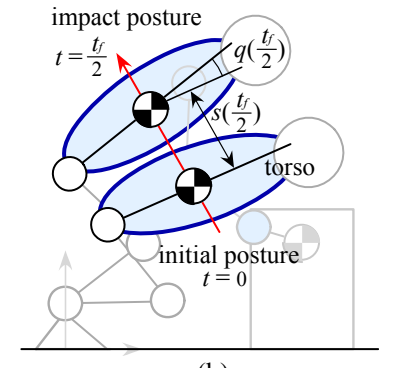

(b)
Fig. 12. Trajectory of the torso. (a) Path. (b) Initial/impact posture.

We design the trajectory of the CoMrob along the path by using a function of the modified constant velocity, which is one of the cam curves [12], [13]. " $S_{c}$ ", " $V_{c}$ " and " $A_{c}$ " in Fig. 13 represent displacement, velocity and acceleration with respect to dimensionless time T. The $S_{c}$ of this cam curve changes from 0 to 1 smoothly as shown in Fig. 13. We use the first half of the cam curve $(0<\mathrm{T}<0.5)$ for design of the motion of the CoMrob.
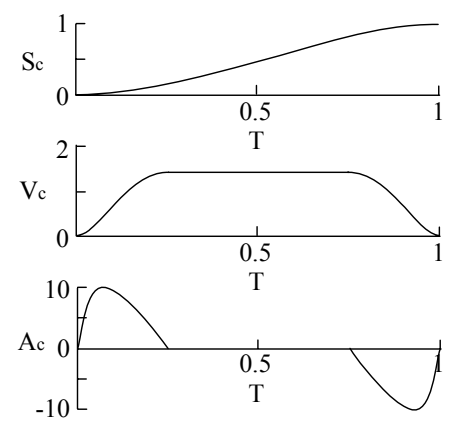

Fig. 13. Cam curve.

Supposing that $t_{f}$ is the finish time of the cam curve in Fig. 13, configuration of torso of robot can be described by path length $s(t)$ and angle $q(t)$ which are given by followings:

$$
s(t)=\alpha S_{c}\left(t / t_{f}\right), \quad q(t)=\beta S_{c}\left(t / t_{f}\right)
$$

where $\alpha$ and $\beta$ are the maximum displacement. Letting $v(t)$ and $\omega(t)$ as derivative of $s(t)$ and $q(t)$ respectively, $v(t)$ and $\omega(t)$ are represented by

$$
v(t)=\alpha / t_{f} V_{c}\left(t / t_{f}\right), \quad \omega(t)=\beta / t_{f} V_{c}\left(t / t_{f}\right)
$$

When impact time $t$ is set as $\mathrm{T}=t / t_{f}=0.5, t=t_{f} / 2$. Then velocities of torso at the impact time can be given by

$$
v\left(t_{f} / 2\right)=* v_{r o b z}, \quad \omega\left(t_{f} / 2\right)=* \omega r o b
$$

Letting $\left|\boldsymbol{r}_{D O D}\right|$ be the distance between $\mathrm{D}_{0}$ and $\mathrm{D},\left|\boldsymbol{r}_{D O D}\right|$ is equal to $s\left(t_{f} / 2\right)$. Then the following equation is given by (9).

$$
\alpha=s\left(t_{f} / 2\right) / S_{c}(1 / 2)=\left|\boldsymbol{r}_{D O D}\right| / S_{c}(1 / 2)
$$

Then $* v_{r o b z}$ is given by (10), (11), and (12) as follows:

$$
*_{\text {robz }}=v\left(t_{f} / 2\right)=\alpha / t_{f} V_{c}(1 / 2)=\left|\boldsymbol{r}_{D O D}\right| / t_{f} V_{c}(1 / 2) / S_{c}(1 / 2)
$$

Since $* \omega r o b$ is given by substituting (8) and (13) to (3), $\beta$ is also calculated by

$$
\beta=\omega\left(t_{f} / 2\right) t_{f} / V_{c}(1 / 2)=* \omega r o b t_{f} / V_{c}(1 / 2)
$$

When the position of the object, the foot of the robot, the initial configuration of the CoM of the robot, the direction of lifting the object and the finish time are given, we can design a trajectory of the torso of the robot in preliminary motion by substituting (12) and (14) to (9).

\section{Constraints}

As shown in Fig. 3, the robot can expand its support area from the foot area $S_{h}-S_{t}$ to the foot-object area $S_{h}-S_{b}$ by holding the object on the floor. Hence the robot can accelerate/decelerate the raising motion, maintaining its stability while object is still on the floor. But there are 
possibilities of slipping, leaning or hoping of the object in this phase because the object is not fixed on the floor. Constraints of a force of the hand to avoid these possibilities are given by following inequalities:

$$
\begin{gathered}
f_{h x}<\mu_{o b j}\left(m_{o b j} g-f_{h z}\right) \\
\tau_{e d g l}=\tau_{h}-m_{o b j} g x_{o b j}+f_{h z} x_{h}-f_{h x} z_{h}<0 \\
\tau_{e d g 2}=\tau_{h}+m_{o b j} g\left(d_{o b j}-x_{o b j}\right)-f_{h z}\left(d_{o b j}-x_{h}\right)-f_{h x} z_{h}>0 \\
m_{o b j} g-f_{h z}>0
\end{gathered}
$$

where $\mu_{o b j}$ is the coefficient of static friction between the object and the floor, $\tau_{\text {edgl }}$ and $\tau_{\text {edg } 2}$ are the torque around the each edge of the object in contact with the floor as shown in Fig. 3. Equations (15), (16), (17), and (18) describe conditions that the object does not slip, lean backward, lean forward, and leave from the floor, respectively.

Furthermore, we denote the coefficient of friction between the floor and the foot of the robot as $\mu_{\text {foot }}$. We have to impose some constraints so that the robot can not tumble or get off the ground. These constraints are written as follow:

$$
\begin{gathered}
f_{\text {footx }}<-\mu_{\text {foot }} f_{\text {footz }} \\
f_{\text {footz }}<0
\end{gathered}
$$

In addition, the ZMP of whole system should be inside the support polygon which is formed by the edge of the robot foot and the object.

\section{Simulation}

We performed simulation through the dynamic simulator developed for the humanoid robot HRP-2 to confirm the effectiveness of the proposed method. We take a box as object. We here assume that grasping point is the CoM of the box, and it has coordinate $(0.54[\mathrm{~m}], 0.495[\mathrm{~m}])$. Mass of the box is $4.5[\mathrm{~kg}]$.

First, we examine the operational force of the hand $f_{h}=\left[f_{h x}\right.$, $\left.f_{h z}\right]^{\mathrm{T}}$ which prevents the box form slipping, leaning and hoping. A curve in Fig. 14 shows that operational force of the hand applied to the box in preliminary motion (from leaning forward to raising upper body). Red area in Fig. 14 represents area of operational force of the hand satisfied with inequalities (15)-(18) where $z_{h}=0.495[\mathrm{~m}], x_{o b j}=0.15[\mathrm{~m}], d_{o b j}$ $=0.3[\mathrm{~m}]$, and $\mu_{o b j}=0.4$. It can be said that the operational force does not move the box in preliminary motion because the curve in Fig. 14 is inside red area. We confirmed that $\boldsymbol{f}_{\text {foot }}$ is satisfied with the condition formed by inequalities (19) and (20).

Second, we show the change of the ZMP with time in Fig. 15. Three broken lines in order from top of Fig. 15 represent X coordinates of point $S_{b}, S_{t}$ and $S_{h}$ in Fig. 3 respectively. Dashed line in Fig. 15 represents impact time which is the start time of lifting motion. As shown in the figure, the ZMP stays in the area between edge of the box and heel of the robot before impact, and it stays in the area of foot after impact. Moreover change of the ZMP for impact is very small. Consequently it is shown that it is possible to lift the box stably by the proposed method.

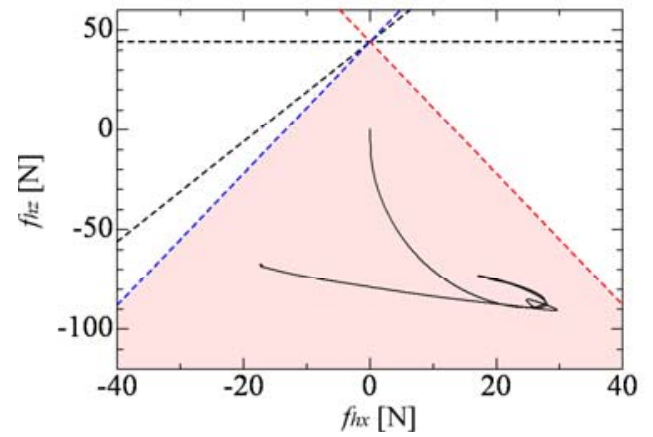

Fig. 14. Operational force to the box.

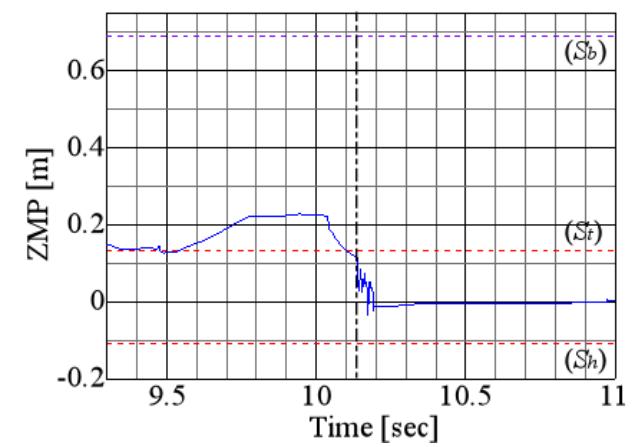

Fig. 15. Simulation result of the ZMP position of the whole system at the impact (broken lines: support area).

Finally animation of lifting motion is shown in Fig. 16. As shown in the figure, the robot can pass through the statically unstable state to the final static stable state without tumbling.

\section{EXPERIMENT}

We performed experiments of the lifting motion by using the humanoid robot HRP-2. The total d.o.f. of HRP-2 is 30 . Also, the height and the weight of the HRP-2 are $\mathrm{h}=$ $1.539[\mathrm{~m}]$ and $m_{\text {rob }}=58[\mathrm{~kg}]$, respectively. Fig. 17 shows change of the ZMP with respect to time. In HRP-2 system, the ZMP can be measured by the force sensors equipped at the foot. But an exact ZMP can not be obtained only by the foot force sensor in the case that the robot contacts the environment through an object such as the experiment. The exact ZMP can be measured when the object leaves the floor. As shown in the Fig. 17, the box is lifted at 10.12[s]. Therefore the data from the time is available. Comparing to simulation results, the direction of change of the ZMP is different, but it is apparent that variations of the ZMP could be reduced.

Fig. 18 shows the snap-shot of the experiment. The robot first squats down. Then, it grasps a box. It further lifts up the box. In the experiment, the weight of the carried box was set as $4.5[\mathrm{~kg}]$. Repeatability of the experiment was confirmed.

\section{CONCLUSION}

In this paper, lifting an object by making use of preliminary motion was discussed. In order to extend capabilities of humanoid robots, we investigated methods for manipulating objects based on preliminary motion. Firstly, we presented a 
method of lifting an object without leaning, tumbling and leaving from floor by using impulsive forces combined with preliminary motion. Secondly, we proposed a method for setting a desired motion allowing the center of percussion to agree with the center of the support polygon to reduce the impact effect received by the robot while lifting the object. The possibility of lifting an object by a humanoid robot has been verified by dynamic simulation and experiments.

A future work is to develop the algorithm to decide a suitable posture of the robot for impact lifting.

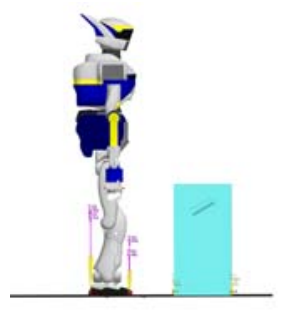

standing

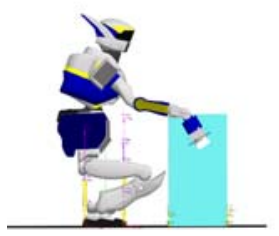

sitting

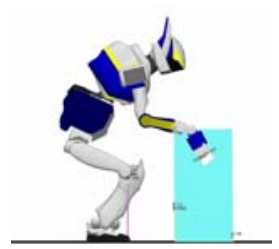

raising

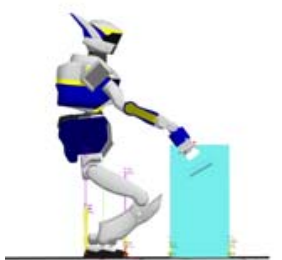

half sitting

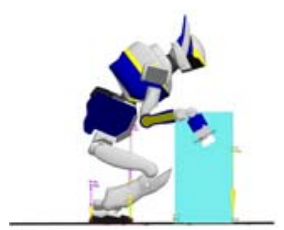

leaning forward

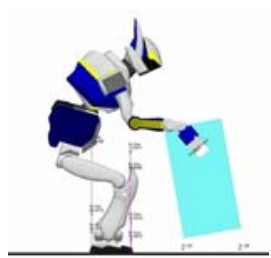

holding
Fig. 16. Lifting motion by HRP-2.

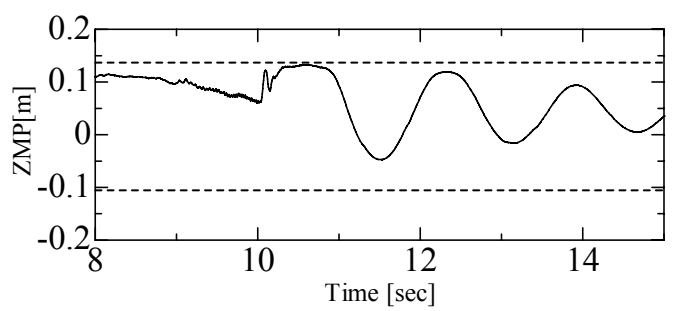

Fig. 17. Experimental result of the ZMP position.

\section{REFERENCES}

[1] H. Arai and O. Khatib, "Experiments with Dynamic Skills,” Proc. 1994 Japan-USA Symposium on Flexible Automation, pp.81-84, Kobe, Japan, 1994.

[2] K. M. Lynch, N. Shiroma, H. Arai and K. Tanie, "The Roles of Shape and Motion in Dynamic Manipulation: the Butterfly Example," Proc. 1998 IEEE International Conference on Robotics and Automation (ICRA'98), pp.1958-1963, 1998.

[3] C. Zhu, Y. Aiyama, T. Chawanya, and T. Arai, "Releasing Manipulation," Proc. IEEE/RSJ Int. Conf. on Intelligent Robots and Systems (IROS'96), pp.911-916, 1996.
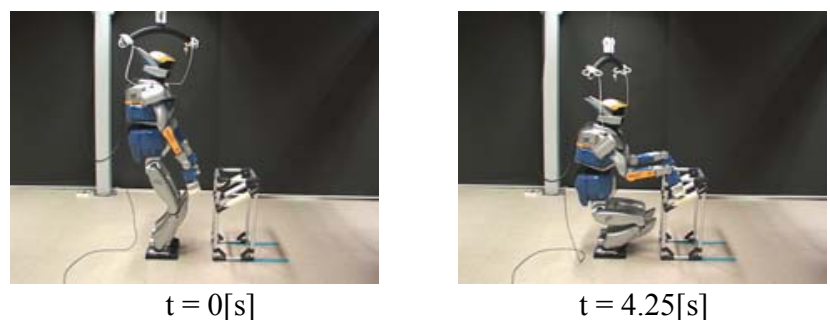

$\mathrm{t}=4.25[\mathrm{~s}]$

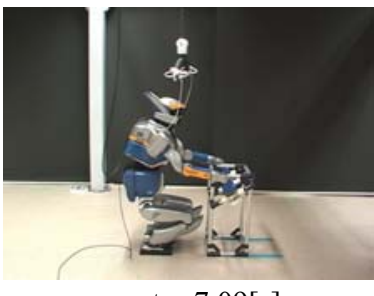

$\mathrm{t}=7.09[\mathrm{~s}]$

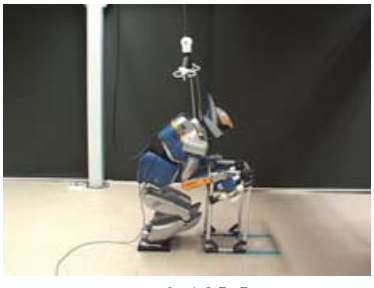

$\mathrm{t}=9.12[\mathrm{~s}]$
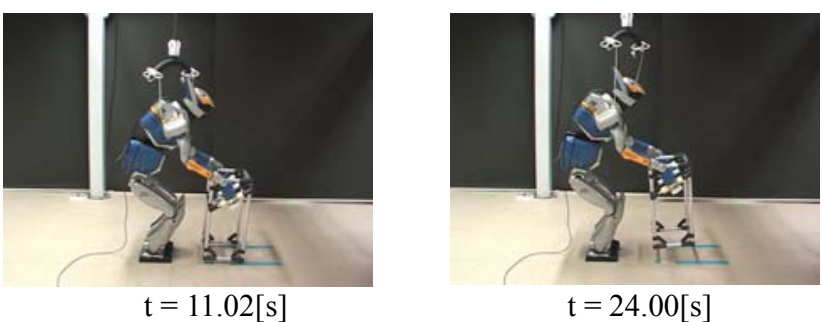

Fig. 18. Snap-shot of experiment.

[4] K. Yokoyama, H. Handa, T. Isozumi, Y. Fukase, K. Kaneko, F Kanehiro, Y. Kawai, F. Tomita, and H. Hirukawa, "Cooperative Works by a Human and a Humanoid Robot," Proc. of IEEE International Conference on Robotics and Automation (ICRA'03), pp. 2985-2991, 2003.

[5] T. Takubo, K. Inoue and T. Arai, "Pushing an Object Considering the Hand Reflect Forces by Humanoid Robot in Dynamic Walking," Proc. of IEEE Int. Conf. on Robotics and Automation, pp.1718-1723, 2005

[6] K. Harada, S. Kajita, K. Kaneko, and H. Hirukawa, "Pushing Manipulation by Humanoid considering Two-Kinds of ZMPs," Proc. of 2003 IEEE International Conference on Robotics and Automation (ICRA'03), pp.1627-1632, 2003.

[7] K. Harada, S. Kajita, H. Saito, M. Morisawa, F. Kanehiro, K.Fujiwara, K. Kaneko, and H. Hirukawa, "A Humanoid Robot Carrying a Heavy Object," Proc. of IEEE Int. Conf. on Robotics and Automation, pp. 1724-1729, 2005

[8] Konno, A., Hwang, Y., Tamada, S. and Uchiyama, M., "Working Postures for Humanoid Robots to Generate Large Manipulation Force," Proc. IEEE/RSJ Int. Conf. on Intelligent Robots and Systems (IROS'05), pp.1788-1793, 2005.

[9] R. Isaka, R. J. Gregor and S. Kawamura, "Derivation of Reasonable Lifting Movement from Techniques Utilized in Skilled Weightlifter," Transactions of the Japan Society of Mechanical Engineers, vol.64, no.623, pp.119-126, 1998.

[10] N. Matsunaga and S. Kawaji, "Motion Analysis of Human Lifting Works with Heavy Objects," Journal of Robotics and Mechatronics, vol.17, no.6, pp.628-635, 2005.

[11] e.g., http://en.wikipedia.org/wiki/Center_of_percussion

[12] Arisumi, H., Yokoi, K., Komoriya, K., "Dynamic Performance Characterization of Manipulators under Actuator Constraints," Proc. of the International Conference on Recent Advances in Mechatronics (ICRAM'95), pp.1042-1047, 1995

[13] Wen-Teng Cheng, "Synthesis of Universal Motion Curves in Generalized Model," Journal of Mechanical Design, Volume 124, Issue 2, pp. 284-293, 2002 